\title{
Causas de la escasa presencia de los deportes de lucha con agarre en las clases de educación física en la ESO. Propuesta de aplicación The causes of the scarce presence combat sport with grip in physical education classes
in compulsory secondary education. Extension proposal
}

\author{
José Robles Rodríguez \\ Universidad de Huelva (España) \\ Resumen: Los deportes suelen ser, en ocasiones, los contenidos más utilizados en las clases de educación física, sin embargo, los deportes de lucha con \\ agarre tienen poca presencia en las programaciones planteadas por los docentes.En el presente artículo analizamos cuáles son las causas de la escasa \\ presencia de estos deportes en secundaria, además de plantear una serie de actividades con las que cualquier docente independientemente de su formación \\ y experiencia previa, podrá poner en práctica los deportes de lucha con agarre en las clases de educación física. \\ Palabras clave: Deportes de lucha con agarre, educación física, iniciación a los deportes de lucha
}

\begin{abstract}
Sports are usually the mostly worked contents in physical education classes. However, combat sport with grip has little presence in teaching planning.In this article, we will analyse which are the causes of the scarce presence of these sports in Secondary Education. In addition, we will propose a sequence of activities which any teacher; regardless his / her training or previous experience, will be able to practise these sports in physical education classes.
\end{abstract}

Key words: Combat sport with grip, physical education, initiation to combat sport with grip.

\section{Introducción}

En la actualidad los contenidos deportivos suelen ser, en la mayoría de los casos, el contenido estrella en las clases de educación física, y así lo corroboran los estudios realizado por Salina y Viciana (2006), Robles (2005), Matanin y Collier (2003), Zabala, Viciana y Lozano (2002), Zabala y otros (2001) y Napper-Owen y otros (1999), donde se observa que el bloque de contenidos de juegos y deportes es el adquiere mayor importancia en las programaciones que los docentes llevan a cabo. Sin embargo, en la mayoría de los casos los deportes de lucha con agarre brillan por su ausencia.

En el presente artículo analizamos las posibles causas de la poca presencia de estos deportes en las clases de educación física. A continuación, planteamos una serie de actividades con las que cualquier docente, independientemente de su formación y experiencia previa, podrá aplicar este contenido deportivo, que seguramente resultará una actividad novedosa, atractiva y muy motivante para los alumnos/as, con el que se contribuirá al desarrollo integral.

Con esta propuesta, pretendemos que cualquier docente tenga la oportunidad de adaptar las actividades de lucha al contexto educativo. En este sentido, Torres y Castarlenas (2004), refiriéndose al judo como deporte de lucha con agarre, afirman que estos, pueden y deben evolucionar desde la perspectiva de la educación física para que la mayoría de la población tenga acceso a su práctica.

\section{EI deporte en el currículo de la ESO}

Hoy en día, podemos considerar el deporte como un fenómeno cultural en nuestra sociedad. En este sentido, el deporte escolar, tanto dentro como fuera de las clases de educación física, el cual debe aparecer siempre asociado a unos valores, actitudes y hábitos que contribuyen al proceso educativo, no puede ser considerado de otro modo. Esta manifestación del deporte ha adquirido gran importancia acaparando el interés de

Fecha recepción: 01-02-08 - Fecha envío revisores: 12-02-08 - Fecha de aceptación: 10-06-08 Correspondencia: Antonio Granero Gallegos

$\mathrm{C} /$ Berrocal, $10,1^{\circ}$

Canino - Huclva (Esp

E-mail: jose.robles@dempc.uhu.es muchas de las instituciones, y una muestra que lo constata ha sido la proclamación por parte del Parlamento Europeo, del «Año Europeo de la Educación a través del Deporte 2004» (Orts, 2004).

El deporte como un contenido dentro de la formación del alumnado de la E.S.O. es utilizado como instrumento educativo que contribuye al desarrollo del currículum de dicha etapa. Para ello, la aplicación de cualquier contenido deportivo en las clases de educación física en todo momento debe cumplir una serie de características. Siguiendo a autores como Le Boulch (1991), Saura (1996), Fraile (1997 y 1999), Feu (2000), Vizcarra y González (2004) entre otros, resaltamos las siguientes características:

- Desarrollar valores y actitudes positivas vinculadas con la práctica deportiva.

- Contribuir al desarrollar las cualidades físicas, afectivoemocionales, cognitivas y sociales acordes a su etapa evolutiva.

- Fomentar hábitos higiénicos y crear hábitos de práctica deportiva en general.

- Debe primar la participación, la cooperación sobre la competición

- Los deportes deben adaptarse a las características de los alumnos/ as y no viceversa.

- Ha de ofrecer diversión y placer en la práctica.

Debemos ser conscientes, en todo momento, de que el sistema educativo se rige en gran medida por lo que demanda la sociedad y, en cierto modo, trata de paliar esas necesidades a través de las propuestas educativas que se desarrollan en los centros escolares. Actualmente, nos encontramos ante una sociedad en la que la demanda de la actividades físico - deportivas tiene gran importancia, de ahí que éste sea uno de los principales motivos por el que el deporte ha de estar presente en el currículum educativo.

Por otro lado, es comúnmente conocido que el deporte aporta beneficios saludables, previniendo al niño de lesiones provocadas por el sedentarismo, guiándole para que ocupen convenientemente su tiempo de libre y de ocio, apartándole del mundo de las drogas, el alcohol, etc....

Con la puesta en práctica de los deportes de lucha contribuiremos al desarrollo de las capacidades propuestas en la ley educativa. Además, favoreceremos que el sujeto practique un nuevo contenido deportivo. 
Queremos resaltar que la UNESCO considera los deportes lucha con agarre, concretamente el judo, un deporte eminentemente formativo, señalando además que los valores que posee lo hacen aconsejable tanto para niños como para adolescentes.

Y para concluir dicho apartado, somos de la opinión de que el deporte hoy en día se ha convertido en un factor muy importante que contribuye al desarrollo de la personalidad del alumno/a y, en este sentido, Bredemeier (1986), citado por Cruz y Col. (1996), afirma que "algunos psicólogos del deporte han señalado que determinadas prácticas deportivas proporcionan un entorno positivo para la socialización de niños y adolescentes, ...», como puede ser el caso de los deportes de lucha con agarre.

3. Causas de la escasa presencia de los deportes de lucha con agarre en las clases de Educación Física

Haciendo referencia a Sánchez Bañuelos (2002), hemos de decir que existen muchos tipos de práctica deportiva relacionadas con una diversidad de contextos. Esta variedad contextual del deporte conforma su estructura, como un conjunto de manifestaciones sociales. El deporte durante el horario lectivo también va a estar influenciado por los distintos contextos. Por lo tanto, los docentes de educación física serán los máximos responsables de inculcar en el alumnado unas actitudes y valores positivos, hacia la práctica de cualquier modalidad deportiva, aspecto que cada vez adquiere mayor importancia, ya que, los estudios realizados por Serra (2006), Moreno y Cols. (2006), entre otros, coinciden en afirmar que la actitud y el interés de los alumnos hacia la Educación Física y el deportes desciende según se avanza en la etapa de secundaria.

Castejón (2004) afirma que la mayoría de los alumnos/as se encuentran con un filtro, de censura previa, que le impide acceder a ciertos conocimientos. En este mismo sentido, Brousse, Villalón y Molina (1999:187), afirman que, «en la educación física escolar la práctica de un reducido número de deportes, producen una estandarización poco favorable de la completa formación de los alumnos, pues disminuye sus experiencias creativas y espontáneas». Por este motivo, dichos autores proponen la incorporación de los deportes de lucha con agarre en el currículum de educación física, con la finalidad de conseguir una educación física más rica y variada.

Los deportes de lucha formar parte del currículum del área de educación física en la E.S.O., concretamente dentro del bloque de contenidos de juegos y deportes, pero la realidad es que esta modalidad deportiva tiene poca presencia en las clases de educación física a lo largo de la etapa de secundaria. A continuación, analizamos cuáles pueden ser las posibles consecuencias.

\section{A. Formación académica del profesorado:}

El primer aspecto que queremos resaltar es el hecho de que no todos los docentes que imparten clases de educación física poseen los estudios específicos para impartir clase en dicha área, debido a que la actual ley permite que cualquier licenciado, independientemente de su titulación, acceda al cuerpo de profesor de secundaria. Concretamente, según los estudios realizados por McCaughtry y otros (2006), Llamas y Moreno (2004), Robles (2005), Viciana y Requena (2002), se observa que entre el 17-28\% de los docentes no poseen estudios de educación física. Por este motivo, podemos afirmar que un porcentaje de los profesores no han recibido formación universitaria relacionada con los deportes y menos aun con los deportes de lucha. En este sentido, Brousse, Villalón y Molina (1999), consideran que la falta de formación de los profesores ha condicionado en gran medida la poca presencia de estos deportes en las clases de educación física.

Por tanto, la solución de a este problema recae de lleno en las administraciones públicas, ya que, mientras que se permita acceder al colectivos de docentes de educación física a profesores con otras licenciaturas, nos encontraremos a un profesorado con poca formación específica.

\section{B. Experiencias e intereses del profesorado:}

En segundo lugar, y basándonos en la afirmación de Castejón y Cols. (2001), las planificaciones realizadas por los docentes tendrán una u otra inclinación en función del dominio de éste. Por ello, y como se indico anteriormente, Castejón (2004), sostiene que un gran porcentaje de los alumnos/as encuentran un filtro, que le impiden acceder a ciertos conocimientos. De ahí, según comenta este mismo autor en el 2005, la selección de los contenidos deportivos viene condicionada por las creencias y por el dominio que el profesor tenga sobre los deportes. Según los datos obtenidos por Robles (2005) y Prat (1999), la mayoría de los profesores han tenido o tienen experiencias como deportistas, sin embargo, son los menos los que han practicado deportes de lucha con agarre.

Según los estudios realizados por Aparecida y Ferreira (2004); Matanin y Collier (2003); Zabala, Viciana y Lozano (2002); Napper-Owen y otros (1999), los deportes colectivos son los más practicados en las clases de educación física en detrimento de los deportes de lucha con agarre.

En este sentido, podemos decir que son las facultades en donde se forman a los profesionales de educación física las que pueden subsanar, en cierto modo, este problema. Para ello, se debería incluir en sus programas, no sólo la enseñanza de los deportes de lucha como tal, sino darle un nuevo matiz a la asignatura, en donde parte de la misma esté dedicada al estudio de la puesta en práctica de los deportes de lucha con agarre dentro del aula.

C. Instalaciones y materiales de que dispone el centro educativo:

Otro factor que debemos considerar es el porcentaje de los docentes que imparten los contenidos deportivos en función de las instalaciones y los materiales que poseen en el centro educativo (Robles, 2005; Viciana y otros, 2004; Viciana y Requena, 2002). En este sentido, Castejón (2004) afirma que las instalaciones deportivas en los centros educativos están construidas para la práctica de determinados deportes tradicionales como el fútbol, baloncesto, balonmano.

Brousse, Villalón y Molina (1999), en referencia a los deportes de lucha, afirman que el no disponer de instalaciones específicas para estas modalidades deportivas, acentúa que en nuestro país no se suelan incluir en las clases de educación física. Pocos son los centros en los que nos encontramos instalaciones adecuadas para la práctica de los deportes de lucha, aunque desde aquí, creemos que no debe ser éste unos de los aspectos que limite la puesta en práctica de estos deportes.

\section{Demandas e intereses del alumnado:}

Siguiendo con los aspectos que los docentes tienen en cuenta a la hora de plantear los contenidos deportivos, las demandas e intereses del alumnado es uno de los factores más importantes que debe regir la planificación (Viciana, 2002), aspectos que tiene en cuenta un alto porcentaje del profesorado (Salina y Cols., 2006). En este sentido, los alumnos/as actualmente reciben muy pocos estímulos relacionados con la práctica de los deportes de lucha con agarre, comparados con los que puede recibir en relación con otras disciplinas deportivas desde los medios de comunicación, las organizaciones deportivas y, fundamentalmente por parte de los padres, quienes, según Carratalá y Carratalá (2000), tienen gran importancia como agentes de socialización, ya que existe una gran correlación entre los intereses de los padres hacia el deporte, y los niveles de participación deportiva de los hijos.

\section{E. Escasez de propuestas didácticas:}

En la literatura específica, nos encontramos con que las propuestas didácticas, en relación con los deportes de lucha con 


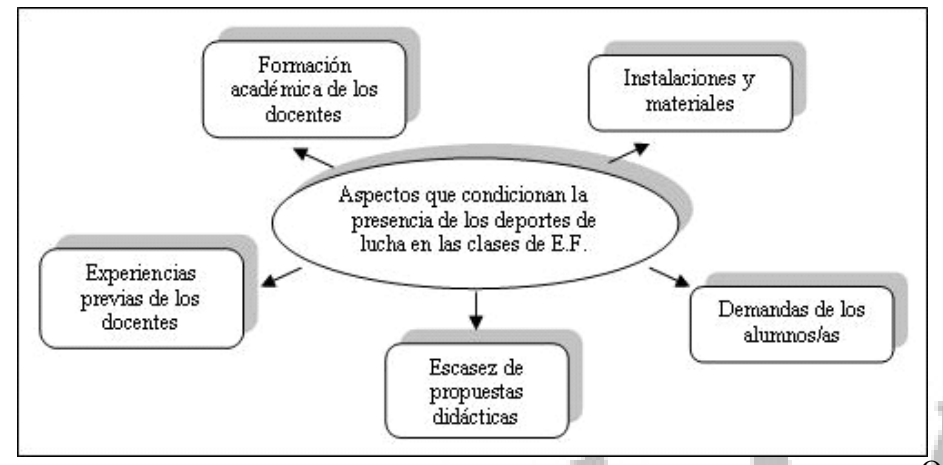

Dentro de los principios estratégico-tácticos de los deportes de lucha con agarre, según Molina y Castarlenas (2002), nos encontramos con proyectar y controlar al adversario. Según el planteamiento que a continuación proponemos nos centraremos en el segundo principio, con la finalidad de tener un mayor control de las actividades. Es por ello, que la mayoría de las actividades que planteamos se desarrollarán en situaciones de lucha en suelo debido a que éstas no plantean problemas especiales de seguridad, siempre y cuando se tenga en cuenta las distintas acciones prohibidas que a continuación exponemos:

Figura 1: Aspectos que condicionan la presencia de los deportes de lucha con agarre en las clases de educación física

agarre que faciliten la puesta en práctica de los mismos desde un modelo educativo-formativo, son muy escasas (Brousse, Villalón y Molina, 1999). Aunque cada vez son más, nos encontramos con que la mayoría de ellas van dirigidas a docentes que en cierta medida conocen o dominan los deportes de lucha con agarre.

Con este trabajo se pretende dar a conocer nuevas formas de trabajar los deportes de lucha en las clases de educación física, sin la necesidad de tener conocimientos específicos sobre este contenido.

Finalmente, una vez presentado los principales aspectos que condicionan la presencia de los deportes de lucha con agarre en las clases de educación física, nos centraremos en las consideraciones metodológica que se deben tener en cuenta para que cualquier docente que imparta la asignatura de educación física pueda incluir en su programación los deportes de lucha con agarre sin la necesidad de haber recibido estudios específicos sobre los mismos.

\section{Consideraciones metodológicas a tener en cuenta}

Los deportes de lucha con agarre, y más concretamente el judo, según Carratalá (2000), se caracteriza por la constante necesidad de adaptarse a los diferentes adversarios y situaciones a la vez que exploramos las acciones con la que nosotros obtenemos un mayor rendimiento. Este último factor nos obliga a elegir entre los múltiples recursos en función de nuestras posibilidades y de las limitaciones del adversario, hecho que exige que el profesor ofrezca una variedad de situaciones de trabajo que desarrollen las capacidades personales del alumno.

Es por este motivo por el que los docentes deben plantear las actividades de lucha de un modo simple y en forma de juego. Este planteamiento facilitará en gran medida la participación de todos los alumnos independientemente de sus características psicofísicas, además de poder comprender mejor la lógica interna de estos deportes. Autores como Villamón y Molina (1999) apuntan que los métodos activos llegan al campo de los deportes de lucha a partir de las teorías constructivitas del aprendizaje, caracterizados principalmente por las experiencias previas de los alumnos/as y para proponer nuevos aprendizajes según esas experiencias, construyendo de ese modo nuevos conocimientos.

La perspectiva de enseñanza que consideramos más oportuna para el desarrollo de las actividades de lucha con agarre se debe basar en los principios propuestos por los métodos activos. Según Devís (1996), estos métodos se caracterizan por que parte de situaciones reales de juego y globales, la variedad de las actividades resulta algo esencial frente a la repetición, además no existen los errores como tales, sino que son respuestas que van evolucionando para dar soluciones a los problemas planteados durante las actividades.
- Queda terminantemente prohibido golpear al compañero. - En ningún caso se podrá tocar la cara al compañero.

- Está prohibido rodear el cuello del compañero con el brazo

- No se podrá empujar hacia atrás al compañero, para evitar la hiperflexión de rodillas.

\section{Propuesta de aplicación}

La propuesta que a continuación se presenta forma parte de la programación didáctica del departamento de educación física del IES Diego Angulo, en Valverde del Camino, Huelva. Dicha propuesta es parte de los contenidos dedicados a los deportes de lucha, los cuales se estructuran en tres unidades didácticas. Una primer unidad didáctica centrada en la iniciación a las actividades de lucha con agarre que se lleva a cabo durante el primer ciclo de secundara, la cual se presenta en dicho artículo. Una segunda unidad didáctica dedicada a la iniciación del judo suelo. Y finalmente, la tercera unidad didáctica se desarrolla en primero de bachillerato, en donde se trabaja sobre el judo pie.

Las actividades que proponemos son juegos de lucha donde se busca fundamentalmente mantener o conseguir la posesión de un objeto, y mantener o conseguir un espacio o una posición, coincidiendo de este modo con los objetivos propuestos por Mansilla y Jurado (2002), para la aplicación del judo en las clases de educación física en la etapa de primaria.

1 A través de la siguiente propuesta vamos a contribuir de manera directa en el desarrollo de tres de los objetivos generales del área de educación física propuestos en el Real Decreto 1631/ 2006, los cuelas nombramos a continuación:

Objetivo $n^{\circ}$ 7. Conocer y realizar actividades deportivas y recreativas individuales, colectivas y de adversario, aplicando los fundamentos reglamentarios, técnicos y tácticos en situaciones de juego, con progresiva autonomía en su ejecución.

Objetivo $n^{\circ} 8$. Mostrar habilidades y actitudes sociales de respeto, trabajo en equipo y deportividad en la participación en actividades, juegos y deportes, independientemente de las diferencias culturales, sociales y de habilidad.

Objetivo $n^{\circ}$ 10. Adoptar una actitud crítica ante el tratamiento del cuerpo, la actividad fisica y el deporte en el contexto social.

Además de los objetivos generales nombrados, a continuación, presentamos objetivos didácticos y los contenidos que vamos a desarrollar mediante el desarrollo de esta propuesta.

\section{Objetivos:}

- Transmitir las características, las normas y los valores propios de los deportes de lucha.

- Participar de manera activa y reflexiva en el desarrollo de las actividades colaborando con el compañero.

- Experimentar y conocer nuevas posibilidades de práctica deportiva mediante los deportes de lucha con agarre.

- Mejorar sus cualidades físicas básicas y sus posibilidades de coordinación y control motor.

- Favorecer las relaciones sociales y el trabajo en equipo mediante la práctica de los deportes de lucha con agarre. 


\section{Actividades cuyo objetivo es mantener o conseguir la posesión de un objeto}

Actividad 1: Por pareja, en pie, ambos sujetando una pelota entre sus manos, intentar quedarse en posesión de la pelota.

Variantes: Comenzar el juego en diferentes posiciones iniciales, sujetando la pelota con una sola mano, con las dos de rodillas.

Actividad 2: Por parejas, agarrados por las mangas, cada uno introduce un pie dentro de un aro, intentar sacar del mismo al compañero.

Variantes: Comenzar con distintos agarres iniciales, a los hombros, una mano al hombro y otra a la manga.

Actividad 3: Por parejas, agarrados por las mangas, intentar colocarse detrás del compañero, es decir, pegar el pecho a la espalda del compañero.

Actividad 4: Por parejas, uno frente a otro de rodillas con una cuerda atada a la cintura, intentar quedarse en posesión de la cuerda del compañero.

Actividad 5: Por parejas, uno frente a otro a dos metros de distancia con una cuerda en medio de ambos. A la señal cada uno intentará coger la cuerda y llevarla a su zona, para evitarlo podemos agarrar al adversario, inmovilizarlo.

Actividad 6: En grupos de cinco ó más. Cada grupo poseen en su campo 5 pelotas. El objetivo es mantener la posesión de estás sin que los adversarios la roben y la lleven a su campo. Para evitarlo podemos inmovilizar a los adversarios. Cambio de rol después de un tiempo determinado.

- Potenciar la cooperación frente a la competición.

- Conocer y experimentar los principios de acción-reacción, aprovechamiento de la fuerza del compañero en beneficio propio.

\section{Contenidos:}

- Conocimiento de las normas básicas de los deportes de lucha con agarre.

- Familiarización con las actividades de lucha

- Los valores de los deportes de lucha.

- Situaciones motrices novedosas para el alumno.

- Actitud de respeto y tolerancia hacia todos los compañeros, rechazando discriminaciones por género, sexo, etc...

- Aceptación por las normas propuestas por el grupo.

- Participación activa en las actividades propuestas.

\section{Temporalización:}

En lo que respecta a la temporalización, hemos de decir que las actividades que presentamos a continuación son actividades propias de la parte fundamental de las prácticas de educación física y, no consideramos que dichas actividades necesiten de una estructura cronológica para su puesta en práctica, por lo que pueden ser estructuradas del modo que el docente crea más oportuno para el desarrollo de sus unidades didácticas.

\section{Conclusiones}

Finalmente, queremos resaltar que la aplicación de los deportes de lucha con agarre dentro de las clases de educación física puede ser llevado a cabo por cualquier profesor independientemente de sus experiencias previas, tanto como deportista o en su formación como docente, sólo requiere de las adaptaciones oportunas según las características del contexto en el que se encuentre.

La introducción de los deportes de lucha con agarre es un contenido propio de la asignatura de Educación Física en Secundaria que pueden resultar una actividad novedosa, atractiva y muy motivante para los alumnos/ as, con el que contribuiremos a su desarrollo integral. En cuanto a la instalación y materiales necesarios para el desarrollo de este contenido, podemos afirmar que no necesita de materiales poco usuales en los centros educativos, ya que sólo es imprescindible el uso de colchonetas, pudiéndose realizar las actividades tanto en la pista como en el gimnasio cubierto.

Actividades cuyo objetivo es mantener o conseguir una posición o un espacio

Actividad 7: Por parejas, ambos intentarán sacar al compañero de la colchoneta, o de una zona delimitada.

Variantes: Comenzar en distintas posiciones (de rodillas, sentado, con agarre de una sola mano, con dos agarres...).

Actividad 8: Por parejas, en pie, ambos agarrados por las caderas intentar levantar al compañero del suelo de manera que no tenga ningún apoyo en el mismo.

Actividad 9: Por parejas, ambos de pies con un agarre predeterminado, intentar sacar al compañero de una zona delimitada.

Variantes: Comenzar con distintos agarres iniciales, a los hombros, una mano al hombro y otra a la manga.

Actividad 10: Por parejas, uno en posición tendido prono o tendido supino y el compañero intenta sacarlo de la colchoneta o de un espacio delimitado. Cambio de rol después de un tiempo determinado o si se consigue el objetivo.

Actividad 11: Por parejas, agarrados de las mangas en posición de rodillas, intentar que el compañero toque el suelo con cualquier parte del cuerpo.

Actividad 12: Ídem al anterior pero ahora intentaremos que el compañero toque el suelo con su espalda. Comenzar desde distintas posiciones iniciales (espalda-espalda, los dos tendido prono....

Actividad 13: Ídem al anterior pero además de intentar que toque con la espalda el suelo se ha intentar mediante la inmovilización que mantenga esta posición guante quince segundos.

Actividad 14: Por parejas, uno en posición de tendido prono. El compañero intentará darle la vuelta y colocarlo en posición de tendido supino.

Actividad 15: Por parejas, uno en cuadrupedia, el otro encima a caballo. A la señal el que está en cuatro apoyos intenta ponerse de pie y el que está encima tendrá que evitarlo.

Actividad 16: Por parejas, uno boca arriba y el compañero sobre éste, en posición de pecho sobre pecho, el que está abajo intenta ponerse boca abajo y el que está arriba ha de evitarlo sin ayuda de los agarres corporales.

Actividad 17: Ídem al anterior pero sin limitación de agarres.

Actividad 18: En grupos de cinco ó más. Un grupo se agarran entre sí formando una piña, los otros cinco deben ir desprendiendo los "piñones" y llevarlos a una zona determinada de antemano.

Actividad 19: En grupos de cinco ó más. Todos formando un corro agarrados de las mangas, intentar caer al suelo a los compañeros a los que se está agarrado mediante barridos de pies. 


\section{Referencias}

Aparecida, D. y Ferreira, P. (2004). Educação física escolar ou esportivização escolar? Lecturas: Educación Física y deportes. Revista Digital, 48. Consultada el 2 julio 2007 en http://www.efdeportes.com/efd78/ escolar.htm

Brousse, M., Villamón, M. y Molina, J. P. (1999). El judo en el contexto escolar. En M. Villamón (Ed.), Introducción al judo (pp. 183-199). Barcelona: Hispano Europea.

Carratalá, E. y Carratalá, H. (2000). Análisis de los motivos de práctica del judo en las distintas federaciones autonómicas. Ponencia presentada en el I Congreso de la Asociación Española de Ciencias del Deporte Facultad de Ciencias del Deporte, Extremadura.

Carratalá, V. (2000). La iniciación al judo, III Jornadas internacionales de Judo. Málaga: Unisport.

Castejón, F. J. (2004). Una aproximación a la utilización del deporte en la educación. Lecturas: Educación Física y deportes. Revista Digital, 73. Consultada el 12 junio 2007, en http://www.efdeportes.com/efd73/deporte.htm

Castejón, F. J., Caro, D., Gamarra, A., Hernando, A., López, I., Nieto, V. y cols. (2001). La evaluación de la programación del profesor y la influencia de su pensamiento en el diseño de la misma. Revista de Educación Física. Renovar la teoría y la práctica (81), 5-12.

Cruz, J., Boixados, M., Torregrosa, M. y Minbrer, J. (1996). ¿Existe un deporte educativo?: papel de las competiciones en el proceso de socialización del niño. Revista de psicología del deporte (9-10), 111-132.

Devís, J. (1996). Educación física, deporte y currículum. Investigación y desarrollo curricular. Madrid: Visor.

Feu, S. (2000). Las actividades extraescolares en la escuela primaria. Una propuesta para llevar los programas de las Escuelas Deportivas a los Centros Escolares. En Actas del I Congreso Nacional de Deporte en edad escolar (pp. 323335). Sevilla: Ayuntamiento de Dos Hermanas.

Fraile, A. (1997). Reflexiones sobre la presencia del deporte en la escuela. Revista de Educación Física (64), 5-10.

Fraile, A. (1999). Perspectiva crítica de una experiencia de deporte escolar. En II Jornadas Andaluzas sobre la sociología del deporte. Málaga: IAD.

Le Boulch, J. (1991). El deporte educativo. Psicocinética y aprendizaje motor. Barcelona: Paidos.

Llamas, L. y Moreno, J. A. (2004). Perfil docente del profesor de educación física en la enseñanza de habilidades gimnásticas y acrobáticas [Versión electrónica]. Lecturas: Educación Física y deportes. Revista Digital. Consultada el 20 junio $2007 \mathrm{en} \mathrm{http//efdeportes.com/efd78/gimn.htm.}$

Mansilla, M. y Jurado, M. D. (2002). as habilidades de lucha con agarre en la conquista de objetivos y espacios. En J. L. Castarlenas y J. P. Molina (Eds.), El judo en la educación física escolar. Unidades didácticas (pp. 65-88). Barcelona: Hispano Europea.

Matanin, M. y Collier, C. (2003). Longitudinal analysis of preservice teachers' beliefs about teaching physical education. Journal of teaching in physical education, 22, 153-168.

Mccaughtry, N., Barard, S., Martín, J., Shen, B. y Hodges Kulinna, P. (2006). Teachers' Perspectives on the Challenges of Teaching Physical Education in Urban Schools: The Student Emotional Filter Research Quarterly for Exercise and Sport. Washington, Tomo 77 (4), 486-498.

Molina, J. P. y Castarlenas, J. L. (2002). Bases para una propuesta para la enseñanza del judo en el contexto escolar. . En J. L. Castarlenas y J. P. Molina (Eds.), El judo en la educación física escolar. Unidades didácticas (pp. 29-44). Barcelona: Hispano Europea.
Moreno, J. A.; Hellín, P. y Hellín, Mª G. (2006). Pensamiento del alumno sobre la educación física según la edad. Apunts. Educación Física y deportes (85), 28-35.

Napper-Owen, G. E., Kovar, S. K., Ermiler, K. L. y Mehrhof, J. H. (1999). Curricula equity in required ninth-grade physical education. Journal of teaching in physical education (19), 2-21.

Orts, F. (2004). La legislación deportiva en las Comunidades Autónomas y el modelo educativo del deporte. En Actas del III Congreso Nacional de Deporte en Edad Escolar (pp. 309-324). Sevilla: Ayuntamiento de Dos Hermanas.

Prat, M. (1999). El perfil personal y profesional del maestro de Educación Física en Cataluña. En P. Sáenz-López, J. Tierra y M. Díaz (Eds.), Actas del XVII Congreso Nacional de Educación Física, pp. 1435-1445 (Vol. II). Huelva: IAD. Universidad de Huelva.

Robles, J. (2005). El deporte en las clases de educación física en la E.S.O. de la provincia de Huelva. Wanceulen EF Digital. Consultada el 28 junio 2007 en http://www.com/revista/ nos.anteriores/numero1.diciembre05/articulos/articu$10 \% 201-7 . h t m$.

Salina, F. y Viciana, J. (2006). La planificación de los bloques de contenidos de la Educación Física en Educación Secundaria Obligatoria, 3. Consultada el 14 Abril 2007 en http:// cienciaydeporte.net/articulo 0306 1.php

Salinas, F., Miranda, M. T. y Viciana, J. (2006). ¿Hacia donde orientan los profesores de E.F. en su formación inicial sus planificaciones? Comparación entre las facultades de España y Galicia. Revista de Educación Física. Renovar la teoría y la práctica (101), 5-14.

Sánchez Bañuelos, F. (2002). Perspectivas y orientaciones para el deporte en la escuela. Educación y Futuro. Revista de investigación aplicada y experiencias educativas (6), 1127.

Saura, J. El entrenador en el deporte. Barcelona: Institut D'estudis Ilerdencs.

Serra, J. R. (2006). Estudio epidemiológico de los niveles de

1 actividad física en los estudiantes de Educación Física Obligatoria. Apunts. Educación Física y deportes (83), 25-34.

Torres, G. y Castarlenas, J. LL. (2004). Deporte, estilo de vida activo y población: consideraciones a través del judo. Apunts. Educación Física y deportes (75), 32-38.

Viciana, J. (2002). Planificar en Educación Física. Barcelona: Inde.

Viciana, J. y Requena, B. (2002). La planificación de la Educación Física por el profesorado en formación permanente. En J. Viciana, Planificar en Educación Física (pp. 237-252). Barcelona: Inde.

Viciana, J., Zabala, M., Dalmau, J. M., Miranda, M. T. y Sánchez, C. (2004). Análisis de las opiniones del profesorado en formación inicial acerca de la planificación de la educación física. En I Congreso nacional innovación y experiencias educativas en el ámbito de la actividad física y el deporte (pp. 48-59). Jerez de la Frontera (Cádiz).

Villamón, M. y Molina, J. P. (1999). La iniciación deportiva en el judo. En M. Villamón, Introducción al judo (pp. 154165). Barcelona: Hispano Europea.

Vizcarra, M. T. y González, I. (2004). Ejemplificación de una propuesta metodológica para trabajar habilidades sociales en el deporte escolar. En Actas del III Congreso Nacional de Deporte en Edad Escolar (pp. 141-159). Sevilla.: Ayuntamiento de Dos Hermanas.

Zabala, M., Viciana, J. y Lozano, L. La planificación de los deportes en la educación física de E.S.O. Lecturas: Educación Física y deportes. Revista Digital, 48. Consultada el 2 julio 2007 en http//efdeportes.com/efd48/eso.htm 